\title{
A Bidding Model for a Virtual Power Plant via Robust Optimization Approach
}

\author{
Geng Tianxiang ${ }^{1}$, Xiang $\mathrm{Li}^{1}$, Ding Maosheng ${ }^{1}$ and Li Feng ${ }^{2}$ \\ ${ }^{1}$ State Grid Ningxia Electric Power Company, 750001 Yinchuan, China \\ ${ }^{2}$ Electric Power Research Institute, State Grid Ningxia Electric Power Company, 750001 Yinchuan, China
}

\begin{abstract}
The evolution of the energy markets has been accelerating the use of distributed energy resources (DERs) all over the world. Virtual power plant (VPP) is a new method to management this increasing two-way complexity. In this paper, a bidding model for a VPP via robust optimization in the uncertain environment of the electricity market is presented. The flexible feature embedded in the model with respect to solution accuracy and computation burden would be advantageous to the VPP. Results of a case study are provided to show the applicability of the proposed bidding model.
\end{abstract}

\section{Introduction}

Due to the global warming and environment concerns of coal-based power generations, the design and operation of the power system are being changed [1-2]. As the penetration of distributed energy resources (DERs) in the distribution network is increasingly grown up, for a smart power system DERs are need to supply power to the grid as much as they could. Moreover, several important issues of these generator units still remain to be solved. Virtual Power Plants (VPPs) are new methods of solving grid-integration of DERs. By integrating these units into VPPs, it can either for the purpose of trading electric energy or to provide support services to the power system.

VPPs can be seen as some conventional dispatchable units in order to compensate for the intermittency of renewable generations by generating technologies. In this sense on the one hand VPPs represents a mixture of multiple DERs and some small-scale conventional power plants. On the other hand, VPPs is operated as one unique entity to participate in the power system. According to FENIX [3], VPPs can be divided into two types, the commercial VPPs (CVPPs) and the technical VPPs (TVPPs). In smart grid, the CVPPs aims to economically optimize its dispatching schedules when trading electrical energy or to provide support services. After that, the CVPPs give the results of the optimal schedules to TVPPs as feedback signals with the consideration of the local network constraints. It should be emphasized that CVPPs only perform commercial aggregation of various kinds of DERs and do not take any network operation constraints into consideration.

At present, the challenges and opportunities in optimal scheduling and bidding strategies in electric markets of VPPs have already discussed in many literatures. In [4-5] the bid-ding strategy of VPPs with centralized control for participating in energy and spinning reserve markets are evaluated. In different cases, the results show the effectiveness and the quality of the proposed model. In [6] the impact of demand response at demand side on power system operation is assessed. Results show that a higher amount of uncontrollable capacity increase these benefits and therefore the social value of demand response. In power system, the centralize dispatch for geographically dispersed DERs will inevitably cause difficulty to the dispatch center. To deal with these problems, to maximize the profit of VPP the coordination of decision-making units embedded in DERs using novel software is proposed in [7]. An energy management system (EMS) of VPPs with a cluster of small-scale generation units, storage systems and flexible loads is proposed in [8]. With the technology of state-ofthe-art communication, a multitude of DERs can be coordinated and controlled by EMS while on the other hand a VPP in reality is not a physical power plant. In EMS, DERs as VPP coalition members can be managed according to its own objectives such as maximization of the total profit of the VPP owner in conjunction with the minimization of the risk of the profit variability. An optimal bidding strategy in the day-ahead market of a microgrid consisting of distributed generation, storage, dispatchable DG and price responsive loads is proposed in [9]. The bidding problem aims to coordinate the energy production and consumption of its components and trade electricity in both day-ahead and real-time markets with the objective to minimize its daily operation cost. A bidding model for a virtual power plant consisting of a wind farm, a pumped storage power plant and three gas turbines considering uncertainties is developed in [10]. In 
the model, VPP aims to maximum its profits when participate in the operation of the mid-term contract market, the day-ahead market and the balancing market. The uncertainty of the electricity price, wind power output and power unbalance penalty are considered in the model.

In conclusion, in the literature, some of worthwhile re-search works investigated specific VPPs in details and elucidates in theory possible benefits of aggregating various kinds of DERs by VPPs. Nevertheless, what is missing in the exist literature is a thorough analysis of the effect of the uncertain-ties in the bidding model for a VPP. Due to the scale of the scenario-based stochastic optimization model increases drastically when the number of the scenarios increase and it will bring huge computational burdens. On this condition, we suggest a RO-based model to deal with the uncertainties in the bidding model.

Based on the above discussion, a bidding model for a VPP via robust optimization approach is proposed. This paper is organized as follows. The VPP bidding problem in a deterministic format and a robust format is established in Section 2; Section 3 presents simulation and results based on examples of IEEE system. Finally, conclusion is drawn in Section 4.

\section{Problem formulation}

\subsection{A deterministic bidding model}

In power system, VPP works as a price taker when it sells electricity to the customers and the excess to the dayahead market at the market price. The VPP bidding problem aims to maximize its bidding profit. The deterministic bidding model is formulated as follows:

$$
\max F=\sum_{t=1}^{T}\left(P_{t}^{\mathrm{D}} \lambda_{t}^{\mathrm{D}}-\sum_{k \in \mathrm{GSP}} \alpha_{k}\left\{\lambda_{t}\right\} P_{k, t}-C_{t}\right)
$$

The objective function (1) to be maximized of a profit function $F$ computed by expected revenues minus VPP operation costs. The revenues contains two parts: the income from selling electricity to the customers and the income/cost from selling/buying electricity to the dayahead market

The amount of hourly electricity exchange between VPP and the day-ahead market is limited to the minimum demand of customers and the capacity of interconnection with the main grid:

$$
\begin{gathered}
P_{k, t} \geq-\min \left\{\max \left\{\begin{array}{l}
0,\left(P^{\mathrm{D}, \max }-\sum_{i \in \mathrm{DG}} P_{i}^{\mathrm{DG}, \min }\right. \\
\left.-\left\{P_{t}^{\mathrm{AW}}\right\}^{\min }\right)
\end{array}\right\}, P_{k}^{\mathrm{S} \max }\right\} \\
P_{k, t} \leq \min \left\{\max \left\{\begin{array}{l}
0,\left(\sum_{i \in \mathrm{DG}} P_{i}^{\mathrm{DG}, \max }+\left\{P_{t}^{\mathrm{AW}}\right\}^{\max }\right. \\
\left.+\bar{e}^{\mathrm{ST}}-P^{\mathrm{D}, \min }\right)
\end{array}\right\}, P_{k}^{\mathrm{S} \max }\right\}
\end{gathered}
$$

where $P_{k}^{\text {Smax }}$ represents the rating for exchanging power with the main grid.
In VPP bidding problem, the power selling to the customers, selling/buying the excess/deficit production to/from the day-ahead market should be constrained by power balance constraint. Thus, the following constrain is considered in the bidding model:

$$
\sum_{i} P_{i, t}+P_{t}^{\mathrm{W}}+\sum_{k \in \mathrm{GSP}} P_{k, t}+P_{t}^{\mathrm{d}}=P_{t}^{\mathrm{D}}+P_{t}^{\mathrm{c}}
$$

The upper and lower power of a gas turbine which is con-trolled under VPP are expressed in (5) and (6) respectively.

$$
\begin{aligned}
& P_{i, t} \leq P_{i}^{\max } v_{i, t} \\
& P_{i}^{\max } v_{i, t} \leq P_{i, t}
\end{aligned}
$$

Power output limits of the gas turbines regarding their ramp rate constraints are formulated as follows:

$$
-r_{i}^{\text {down }} \Delta t \leq P_{i, t}-P_{i, t-1} \leq r_{i}^{\text {up }} \Delta t
$$

Constrains (8) and (9) are necessary to model the start-up and shut-down status of the gas turbines and avoid the simultaneous commitment and decommitment of a unit.

$$
\begin{gathered}
y_{i, t}-z_{i, t}=v_{i, t}-v_{i, t-1} \\
y_{i, t}+z_{i, t} \leq 1
\end{gathered}
$$

The minimum up and down time constraints of the gas turbines are modelled in the constraint (10) to constraint (13).

$$
\begin{gathered}
v_{i, t}-v_{i, t-1} \leq v_{i, t+T U_{i, w}} \\
T U_{i, w}= \begin{cases}w & w \leq \mathrm{MUT}_{i} \\
0 & w>\mathrm{MUT}_{i}\end{cases} \\
v_{i, t-1}-v_{i, t}+v_{i, t+T D_{i, w}} \leq 1
\end{gathered}
$$

Finally, the constraints (14) and (15) are used to denote the technical production capacity of a wind farm:

$$
\begin{gathered}
P_{t}^{\mathrm{W}} \leq\left\{P_{t}^{\mathrm{AW}}\right\} \\
P_{t}^{\mathrm{W}} \geq 0
\end{gathered}
$$

\subsection{A robust bidding model}

To deal with uncertain parameters in the optimization models, there are several methods proposed in the exist literature. These methods can be categorized into three main principal categories: probabilistic methods, possibilistic methods, and hybrid probabilisticpossibilistic [11].

Recently, robust optimization has emerged as an attractive optimization framework. Compared with other frame-work mentioned above, robust optimization can 
reduce the sensitivity of the optimal solution to perturbations in the parameter values. The methods which are proposed to address data uncertainty over the years can be categorized into: (1) Stochastic Programming, and (2) Robust Optimization.

As the only uncertain parameter in the deterministic bid-ding model (1)-(15) is the day-ahead market price and it just appears in the objective function, hereupon, use duality theory the robust form can be formulated as below:

$$
\left\{\begin{array}{l}
\min G=\sum_{t=1}^{T}\left[C_{t}+\frac{1}{2}\left(\bar{\lambda}_{t}+\underline{\lambda}_{t}\right) \sum_{k \in \mathrm{GSP}} \alpha_{k} P_{k, t}-P_{t}^{\mathrm{D}} \lambda_{t}^{\mathrm{D}}\right] \\
\quad+\Gamma v+\sum_{t=1}^{T} \eta_{t} \\
\text { s.t. constraints }(2-15) \\
v+\eta_{t} \geq \frac{1}{2}\left(\bar{\lambda}_{t}+\underline{\lambda}_{t}\right) y_{t} \\
-y_{t} \leq \sum_{k \in \mathrm{GSP}} \alpha_{k} P_{k, t} \leq y_{t} \\
v, \eta_{t}, y_{t} \geq 0
\end{array}\right.
$$

\section{Test examples}

In order to illustrate the performance of the model proposed in the previous section, we present a case study using well-known IEEE 30-bus system including a VPP. The VPP is expected to aggregate and control four gas turbines at buses 26, 29 and a wind farm at bus 29 and a storage system at bus 30 . For simplification of the problem, we suppose that the VPP can trade with the dayahead market through three buses at 26, 29 and 30 (Fig. $1)$.

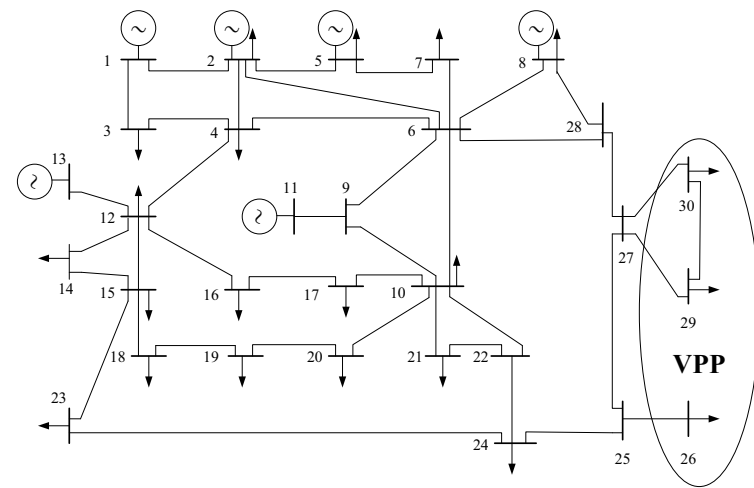

Figure 1. Diagram of the IEEE30-bus containing VPP.

Based on the economic data available in the electricity market of mainland Spain [12], the day-ahead market price forecasts is shown in Table 1. The market prices at the GSPs 26, 29 and 30 are according assumed to be 95,100 , and 105 percent of the day-ahead market prices forecasts. The wind power output forecasts is

\begin{tabular}{|c|c|c|c|c|c|}
\hline$t / \mathbf{h}$ & $P_{t}^{\mathrm{W}} /(\mathbf{M W})$ & $t / \mathbf{h}$ & $P^{\mathrm{W}} /(\mathrm{MW})$ & $t / \mathrm{h}$ & $P^{\mathrm{W}}{ }_{t} /(\mathrm{MW})$ \\
\hline 1 & 39.25 & 9 & 36.91 & 17 & 34.32 \\
\hline 2 & 40.73 & 10 & 34.67 & 18 & 27.85 \\
\hline 3 & 36.63 & 11 & 26.36 & 19 & 22.86 \\
\hline 4 & 43.77 & 12 & 27.42 & 20 & 19.47 \\
\hline 5 & 39.75 & 13 & 33.12 & 21 & 27.30 \\
\hline 6 & 35.10 & 14 & 38.57 & 22 & 31.35 \\
\hline 7 & 30.45 & 15 & 34.56 & 23 & 30.9 \\
\hline 8 & 34.43 & 16 & 34.87 & 24 & 28.17 \\
\hline
\end{tabular}
shown in Table 2.

Table 1. Day-ahead markets price forecasts

\begin{tabular}{|c|c|c|c|c|c|}
\hline $\boldsymbol{t} / \mathbf{h}$ & $\boldsymbol{\lambda}_{\boldsymbol{t}} / \mathbf{( \$ / \mathbf { M W h } )}$ & $\boldsymbol{t} / \mathbf{h}$ & $\boldsymbol{\Lambda} \boldsymbol{t} / \mathbf{( \$ / M W h})$ & $\boldsymbol{t} / \mathbf{h}$ & $\boldsymbol{\lambda}_{\boldsymbol{t}} /(\mathbf{\$} / \mathbf{M W h})$ \\
\hline 1 & 46.03 & 9 & 76.95 & 17 & 108.31 \\
\hline
\end{tabular}

\begin{tabular}{|l|l|l|l|l|l|}
\hline 2 & 45.14 & 10 & 69.09 & 18 & 89.54 \\
\hline 3 & 45.50 & 11 & 65.84 & 19 & 76.83 \\
\hline 4 & 45.70 & 12 & 59.47 & 20 & 73.60 \\
\hline 5 & 55.80 & 13 & 56.47 & 21 & 59.59 \\
\hline 6 & 82.28 & 14 & 53.77 & 22 & 52.47 \\
\hline 7 & 84.80 & 15 & 52.90 & 23 & 47.77 \\
\hline 8 & 83.44 & 16 & 71.44 & 24 & 39.17 \\
\hline
\end{tabular}

Table 2. Wind power output forecasts.

In our case study, two values of parameter $\Gamma$, i.e., two different risk strategies are considered and compared.

Strategy A: A conservative strategy is realized when $\Gamma=24$.

Strategy B: A less conservative strategy is realized when $\Gamma=8$

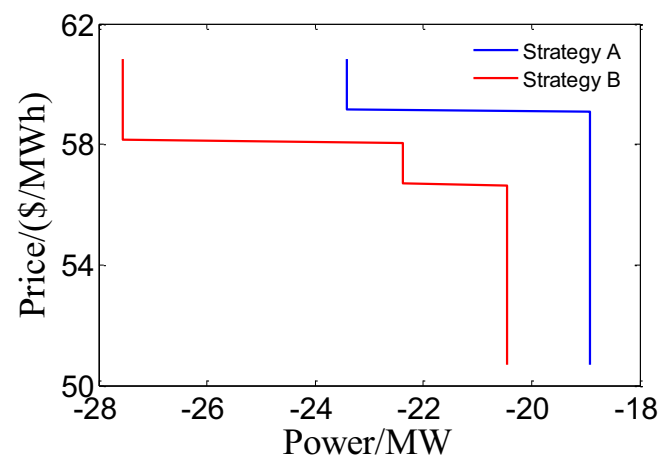

(a)

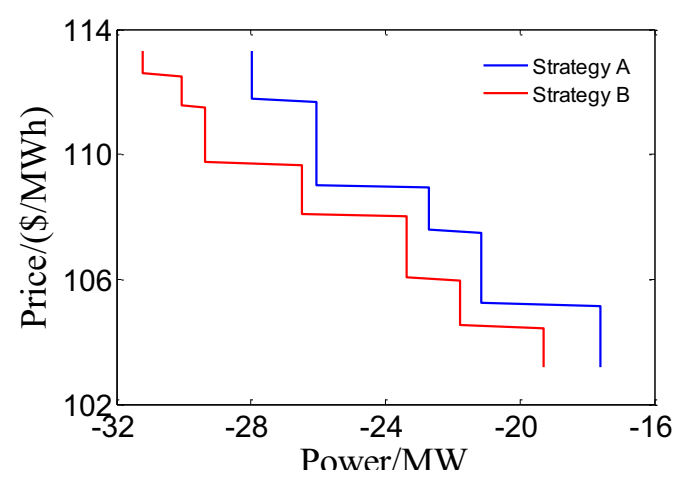

(b)

Figure 2. Bidding curve in the day-ahead market for (a) hour 5 and (b) hour 17.

Fig. 2 depicts the bidding curves in the day-ahead market during hour 5 and hour 17 for the two considered values of parameter $\Gamma$. As is shown in the figure, for a fixed value of parameter $\Gamma$, the willingness to sell energy in hour 17 is higher than in hour 5. For example, using a conservative strategy (Strategy A), the VPP decides to submit the bidding curve within the power internal [-23.4, -18.9] MW in hour 5 while in hour 17 the power internal 
is [-28.0, -17.6] MW. The reason is that in order to increase the bidding profit, VPP decides to sell energy in high day-ahead market price time periods.

Comparison of the results throughout the day indicates that the bidding curve covers a wider power internal in high-price hours than that in low-price hours. It is worthy to note that the bidding curve in each hour moves right by the increase of parameter $\Gamma$. In other words, adopting a less conservative strategy decreases the willing to sell energy in the day-ahead market.

VPP trading with the day-ahead market through the inter-connection grid points is given in Figure 3. It is obvious that the VPP acts as an arbitrager in some hours due to the fact that it purchases energy from the market at the cheapest bus and sells it exclusively to the market at the expensive bus.

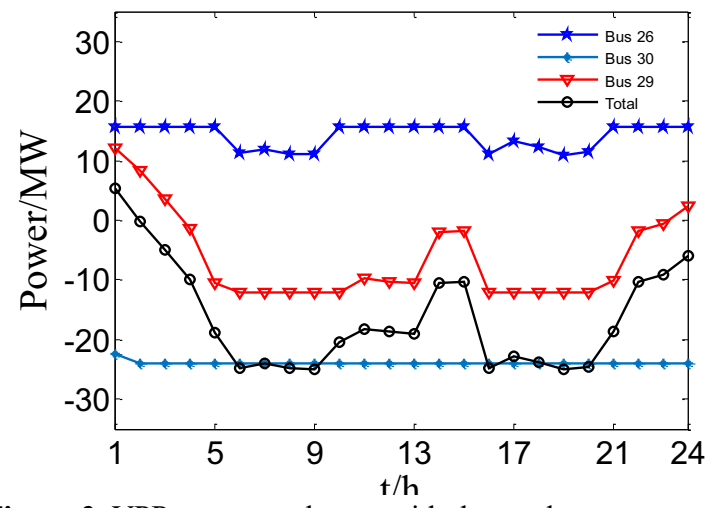

Figure 3. VPP power exchange with the market.

\section{Conclusion}

In this paper, a bidding model for a virtual power plant via robust optimization has been proposed. The proposed robust bidding model guarantees obtaining a maximum bid-ding profit for VPPs provided that the realized dayahead market prices are deviated in a trust region. The advantage of the proposed approach is its flexibility in solution accuracy and computational burden. A case study demonstrates the usefulness and simplicity of the proposed model to maximize the proposed of a VPP considering a wind farm, four gas turbines and a storage system, the conclusions below are in or-der:

1) The proposed bidding model for a VPP via robust optimization allows the VPP to appropriately represent uncertain data.

2) The proposed bidding model allows the VPP to use its DERs to buy and sell energy at suitable according to its objectives.

3) The risk strategy adopted influences the bidding strategy, power traded of a VPP.

\section{References}

1. M. Shabanzadeh, M-K. Sheikh-El-Eslami and M-R. Haghifam, "The design of a risk-hedging tool for virtual power plant via robust optimization approach,” Applied Energy, vol. 155, pp. 766-777, Jul 2015.

2. Z.N. Wei, S. Yu, G.Q. Sun, Y.H. Sun, Y. Yuan and D. Wang, "Concept and development of virtual power plant," Automation of Electric Power Systems, vol. 37, no. 13, pp. 1-9, Jul 2013.

3. D. Pudjianto, C. Ramsay and G. Strbac, "The FENIX vision: The virtual power plant and system integration of distributed energy resources," Contract No: SES6-518272, Deliverable 1.4.0, 2006.

4. E. Mashhour and S.M. Moghaddas-Tafreshi, "Bidding strategy of virtual power plant for participating in energy and spinning reserve markets: Part I Problem Formulation," IEEE Trans on Power Systems, vol. 26, no. 2, pp. 957-964, May 2011.

5. E. Mashhour and S.M. Moghaddas-Tafreshi, "Bidding strategy of virtual power plant for participating in energy and spinning reserve markets: Part II Numerical Analysis," IEEE Trans on Power Systems, vol. 26, no. 2, pp. 957-964, May 2011.

6. B. Dupont, K. Dietrich, C. De Jonghe, A. Ramos and R. Belmans, "Impact of residential demand response on power system operation: a Belgian case study," Applied Energy, vol. 122, pp. 1-10, Jun 2014.

7. H.M. Yang, D.X. Yi, J.H. Zhao and Z.Y. Dong, "Distributed optimal dispatch of virtual power plant via limited communication," IEEE Trans on Power Systems, vol. 28, no. 3, pp. 3511-3512, Aug 2013.

8. P. Lombaedi, M. Powalko and K. Rudion, "Optimal operation of a virtual power plant," 2009 IEEE Power \& Energy Society General Meeting, pp. 1-6, 2009.

9. G.D. Liu, Y. Xu and K. Tomsovic, "Bidding strategy for microgrid in day-ahead market based on hybrid stochastic/robust optimization," IEEE Trans on Smart Grid, vol 7, no. 1, pp. 227-237, Jan 2016.

10. S.Yu, Z.N. Wei, G.Q. Sun, Y.H. Sun and D. Wang, "A bidding model for a virtual power plant considering uncertainties,"Automation of Electric Power Systems, Vol. 38,no. 22,pp. 43-49, Nov 2014.

11. A. Soroudi and T. Amraee, "Decision making under uncertainty in energy systems: State of the art," Renewable and Sustainable Energy Reviews, vol. 28, pp. 376-384, Aug 2013.

12. Iberian Electricity Market Operator, OMIP 2016, $<$ http://www.omip.pt/>. 